\title{
Forest in situ observations using unmanned aerial vehicle as an alternative of terrestrial measurements
}

\author{
Xinlian Liang ${ }^{1} \mathbb{D}$, Yunsheng Wang ${ }^{1 *} \mathbb{D}$, Jiri Pyörälä ${ }^{1}$, Matti Lehtomäki ${ }^{1}$, Xiaowei Yu ${ }^{1}$, Harri Kaartinen ${ }^{1}$, Antero Kukko ${ }^{1}$, \\ Eija Honkavaara', Aimad E. I. Issaoui ${ }^{1}$, Olli Nevalainen ${ }^{1}$, Matti Vaaja ${ }^{2}$, Juho-Pekka Virtanen ${ }^{1,2}$, Masato Katoh ${ }^{3}$ and \\ Songqiu Deng ${ }^{3}$
}

\begin{abstract}
Background: Lately, terrestrial point clouds have drawn attention as a new data source for in situ forest investigations. So far, terrestrial laser scanning (TLS) has the highest data quality among all terrestrial point cloud data in terms of geometric accuracy and level of detail (IEEE Transact Geosci Remote Sens 53: 5117-5132, 2015). The TLS point clouds processed by automated algorithms can provide certain individual tree parameters at close to required accuracy in practical applications. However, all terrestrial point clouds face a general challenge, which is the occlusions of upper tree crowns. An emerging technology called unmanned-aerial-vehicle (UAV) - borne laser scanning (ULS) potentially combines the strengths of above and under canopy surveys.

Results: The performance of ULS are evaluated in 22 sample plots of various forest stand conditions in a boreal forest. The forest parameter estimates are benchmarked through a comparison with state-of-the-art terrestrial mechanisms from both static terrestrial and mobile laser scanning. The results show that in easy forest stand conditions, the performance of ULS point cloud is comparable with the terrestrial solutions.

Conclusions: This study gives the first strict evaluation of ULS in situ observations in varied forest conditions. The study also acts as a benchmarking of available active remote sensing techniques for forest in situ mensuration. The results indicate that the current off-the-shelf ULS has an excellent tree height/tops measurement performance. Although the geometrical accuracy of the ULS data, especially at the stem parts, does not yet reach the level of other terrestrial point clouds, the unbeatable high mobility and fast data acquisition make the ULS a very attractive option in forest investigations.
\end{abstract}

Keywords: In situ, Point cloud, Terrestrial, Mobile, Above canopy, Unmanned aerial vehicle, Forest inventory

\section{Introduction}

Quantitative assessments of forest resources rely on in situ measured parameters (e.g., tree height) of trees and tree communities, which are used as a direct quantificational description of a forest area, or as a reference information for further deduction on regional forest characteristics. In situ measurements play a fundamental role in understanding forest ecosystems and their interactions with other elements in biosphere. In order to represent the diversity

\footnotetext{
* Correspondence: yunsheng.wang@nls.fi

'Department of Remote Sensing and Photogrammetry, Finnish Geospatial Research Institute, 02431 Masala, Finland

Full list of author information is available at the end of the article
}

of forest stands, the measurements are typically carried out in sample plots that are widely and systematically spread over an area of interest. Due to the large size, the structural complexity, the spatial heterogeneity of forests, and the lack of efficient and practical option for forest measurements, precise mensuration of tree parameters with sufficient spatial and temporal resolution are time-consuming and cost-intensive, especially in natural forests. Consequentially, the amount, the frequency, and the parameter richness of in situ forest measurements are generally limited by practical reasons such as the budget and the accessibility in forests. As a matter of fact, the variety of tree parameters, the number of sample plots, as well as the 
spatial and temporal resolutions of in situ measurements are almost always insufficient for further applications. Therefore, all the inferences built on top of the given in situ measurements are potentially biased.

Tremendous efforts have been put into the advancement of hardware, software and measurement protocols to improve measurements of forest sample plots. For example, laser relascope (Kalliovirta et al. 2005) and laser camera (Melkas et al. 2008) were developed to map stem locations and diameter at breast height (DBH) in the field; Photogrammetric methods were proposed to retrieve stem curve of standing trees (Hapca et al. 2007); and panorama images (Dick et al. 2010) were adopted to improve automation of field inventory. Lately, terrestrial point clouds-based studies have presented strong competitiveness for retrieving treeand plot-level forest parameters. In Liang et al. (2018a), it was demonstrated that three-dimensional (3D) forest structure can be measured with a high accuracy and high automation level from point cloud data produced by terrestrial laser scanning (TLS).

Terrestrial point clouds can be recorded directly from laser scanning (LS) systems or indirectly from structured light or highlyoverlapped image sequences. So far, TLS has presented the highest quality among all sources of terrestrial point clouds in terms of the geometric accuracy and the level of details (Liang et al. 2015). TLS uses a direct and stable measurement mechanism. It has been widely understood as the most promising technique in in situ 3D forest digitization, with which tree- and plot-level parameters can be accurately and automatically retrieved for sample plots. Previous research has shown that TLS point clouds processed with automated algorithms can provide certain tree-level parameters with an accuracy that is close to what is required by practical applications (e.g., national forest inventories (NFIs) in boreal forests). For example, the DBH and stem curve can be measured at a root-mean-square -error (RMSE) of 1-2 cm using TLS; The stem volume and the total tree biomass (AGB) can be measured at an accuracy close to $100 \%$ accuracy at plot-level in homogeneous mature forests (Liang et al. 2018a).

Meanwhile, image-based point clouds provide a low-cost alternative to the LS-based point clouds (Liang et al. 2015; Forsman et al. 2016a; Hyyppä et al. 2017; Tomaštík et al. 2017; Mokroš et al. 2018). Forest in situ measurements by non-professional users, such as forest owners, has become feasible by using, e.g., a cellphone camera. Moreover, in recent years, mobile and personal mapping have become available (Liang et al. 2014; Bauwens et al. 2016; Forsman et al. 2016b; Marselis et al. 2016; Juraj et al. 2017; Oveland et al. 2017) and many efforts have been invested to new Mobile Laser Scanning (MLS)-relevant data processing (Kukko et al. 2017; Liang et al. 2018b; Luo et al. 2018). Mobile and personal mapping systems integrating the LS/camera sensors, the kinematic platforms, and/or the navigation sensors, are capable to measure the forest plots 3-20 times faster than stationary systems (Liang et al. 2014). Consequently, the number of the sample plots measured within a time unit may be significantly increased in comparison with the conventional manual and TLS measurements. Furthermore, the size of sample plots can also be significantly larger than what is currently used.

During the last decade, unmanned aerial vehicle (UAV) has become a convenient platform that has increased the automation level of data acquisition and initiated a wide range of applications. Nowadays, the continuous sensor miniaturization had enabled UAV-borne LS (ULS) systems. The advantages of ULS rise from the combination of a near ground aerial perspective for observations and the canopy penetration capacity of the LS sensors. Therefore, ULS avoid the access constrains on the ground and the degradation of Global Navigation Satellite System (GNSS) signals underneath the canopies, which are the main challenges when using terrestrial platforms. In addition, the level of detail of ULS point cloud data is becoming comparable to that of TLS. Thus, the boundary between airborne laser scanning (ALS) and TLS systems has diminished due to the development of ULS, which provides a new type of high-quality point cloud for forest investigations.

Previous studies have understood ULS as a low altitude ALS system, which provides denser point cloud than platforms at high altitudes (e.g., Jaakkola et al. 2010; Wallace et al. 2012, 2014). Tree parameters such as location, height and crown area have generally been measured using individual-tree-based approaches, or metrics like height percentiles in area-based approaches. Though the possibility of measuring the DBH has been noticed (Chisholm et al. 2013; Brede et al. 2017; Jaakkola et al. 2017; Wieser et al. 2017), ULS has not yet been understood as a technology that digitizes forest in a similar manner as the terrestrial systems such as TLS, mobile laser scanning (MLS), personal laser scanning (PLS), and terrestrial image-based point clouds. The question is therefore whether or not the forest can be digitized from above the canopies, and to which extent of accuracy.

This study evaluated the performance of ULS in different forest stand conditions in a boreal forest. For the first time, the ULS point cloud was understood as a technology acquiring terrestrial point clouds in the sense that individual trees were detected using stem detection algorithm and tree parameters were estimated through stem modeling. To differentiate the data- and the processing-level performances, the ULS data were processed using manual measurements and an automated algorithm. The manual and automated estimation accuracy of parameters were evaluated with respect to the field collected reference. For each sample plot, the evaluated parameters include the Digital Terrain Model (DTM), the tree positions, and the $\mathrm{DBH}$, the tree height, the stem curve, the stem volume as 
well as the above-ground biomass (AGB) of each individual tree in the plot. In addition, ULS-based estimates were compared with state-of-the-art TLS and MLS datasets. Findings in this paper are expected to provide a detailed inspection of the ULS data from the perspective of terrestrial point cloud, thus, to clarify the potential of detailed forest digitization from a close-to-ground aerial perspective.

\section{Materials and methods}

The forest sample plots in this experiment represent a variety of stand conditions with regard to species, growth stages and management activities. The forests were measured using four approaches: ULS, MLS/PLS, TLS, and conventional in situ measurements. The performance of ULS was evaluated through the comparison among these observations: the conventional in situ measurements served as a reference, TLS represented the state-of-the-art of automatous terrestrial tree observation approach, and MLS and PLS exemplified emerging solutions with an enhanced mobility and data coverage.

\section{Test area}

The experimental site was established in 2014 and located in a boreal forest in Evo, Finland $\left(61.19^{\circ} \mathrm{N}, 25.11^{\circ} \mathrm{E}\right)$. The main tree species were Scots pine (Pinus sylvestris L.), Norway spruce (Picea abies (H. Karst.) L.), Silver (Betula pendula Roth) and Downy (Betula pubescens Ehrh.) birches. On the site, 24 forest sample plots, $32 \mathrm{~m}$ by $32 \mathrm{~m}$ for each, were selected by foresters to test various in situ measurement approaches.

The sample plots were classified into three stand complexity categories, i.e., "easy", "medium" and "difficult", according to the amount of occlusion at the ground level, the spatial stem density and the distribution of the DBH. The category "easy" represented clear visibility with minimal understory vegetation and low stem density $(\sim 700$ trees/ ha); "medium" represented sample plots with moderate stem densities ( 1000 trees/ha) and sparse understory vegetation; the "difficult" category represented plots with high stem densities ( 2000 trees/ha) and dense understory vegetation.

Figure 1 illustrates tree maps of three example plots from the three different complexity categories. The mean DBH, mean tree height and mean basal areas are $20.5 \mathrm{~cm}, 18.3 \mathrm{~m}$ and $23.1 \mathrm{~m}^{2} \cdot \mathrm{ha}^{-1}$, respectively, for the easy plot (Fig. 1a); $17.3 \mathrm{~cm}, 16.3 \mathrm{~m}$ and $30.2 \mathrm{~m}^{2} \cdot \mathrm{ha}^{-1}$ for the medium plot (Fig. 1b), respectively; and $12.3 \mathrm{~cm}, 13.2 \mathrm{~m}$ and $29.3 \mathrm{~m}^{2} \cdot \mathrm{ha}^{-}$ ${ }^{1}$ for the difficult plot (Fig. 1c), respectively. In general, the mean DBH and tree height decrease and the basal area increases along with the growing complexity of stands.

Figure 2 shows the distribution of the DBH in each stand complexity category by the time of the UAV flight, which illustrates the variation of tree size within each complexity category. As shown by the DBH distribution, the higher the stand complexity, the higher is the population of small trees (e.g., $\mathrm{DBH}<15 \mathrm{~cm}$ ).

In situ observations using conventional field methods

Conventional forest field measurements were carried out between May and August of 2014 (Liang et al. 2018a). All trees with DBH larger than $5 \mathrm{~cm}$ were measured in the sample plots. The tree height and the DBH were measured using conventional field measurement methods, i.e., using calipers and inclinometer. Tree maps were produced for each plot by combining measurements in the field and in the TLS data. Preliminary tree positions were firstly mapped from TLS point clouds. The preliminary tree positions were then verified and updated during in situ investigations, and trees missed on the preliminary tree maps were added. The stem curves were manually digitized from the multi-scan TLS point clouds starting at the height of $0.65 \mathrm{~m}$ above the ground, continuing at the DBH

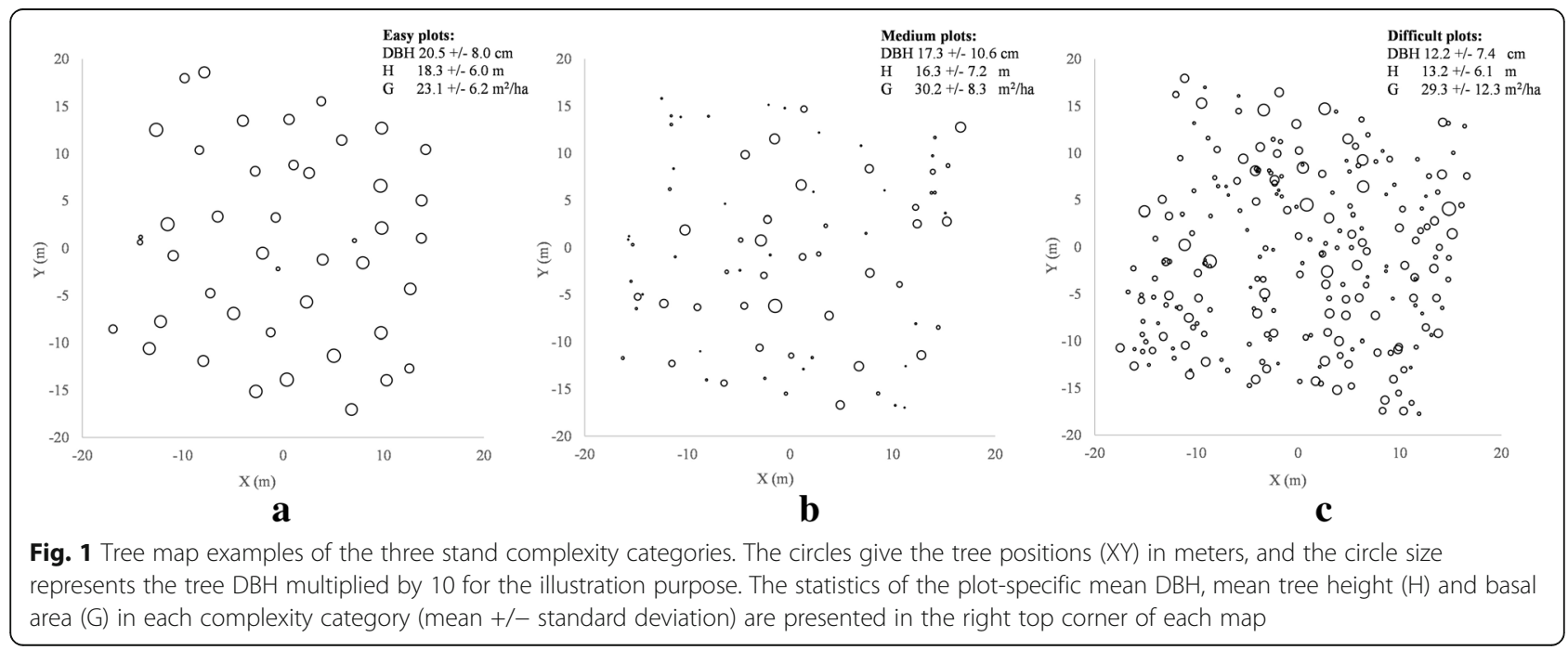




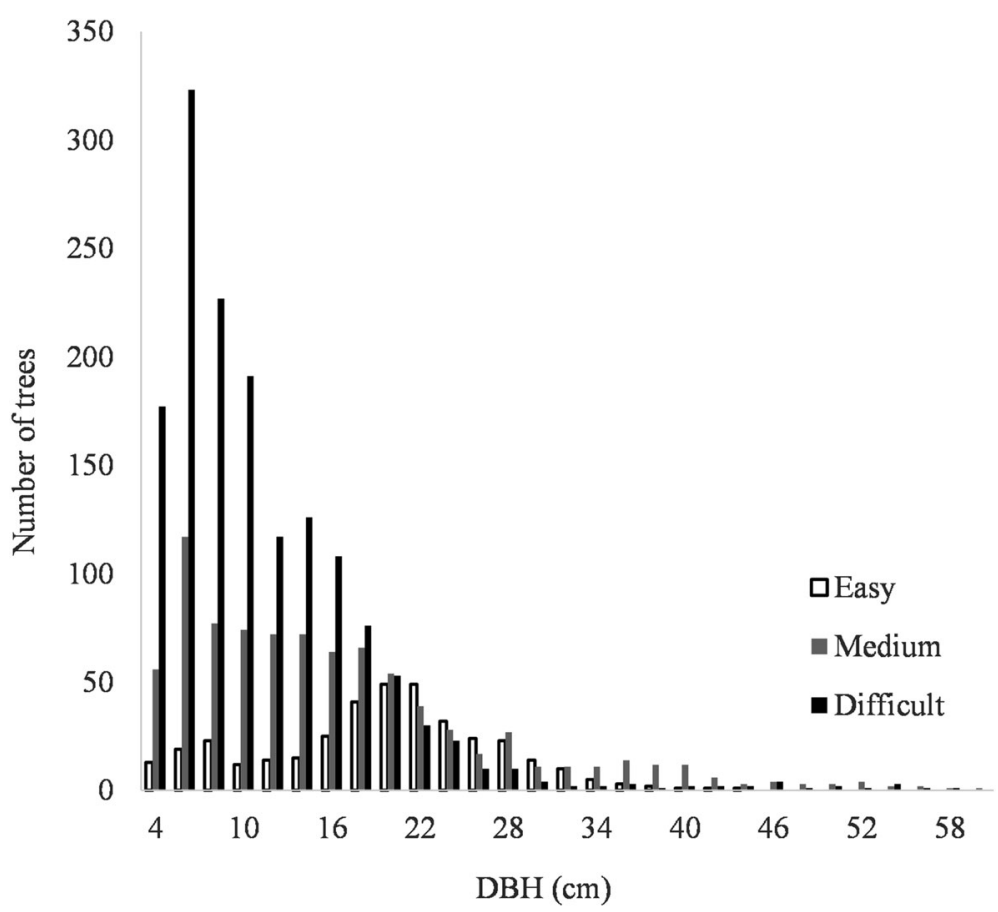

Fig. 2 The distribution of the DBH in each complexity category

height and then every meter above until the maximum measurable height in the point cloud. The stem volume was calculated directly using the tree height and stem curve measurements similarly as in Liang et al. (2018a). The total tree biomass was calculated using Finnish national allometric models (Repola, 2009). The DTM was generated through rasterization of the classified ground points using TerraScan software (TerraSolid Oy, Helsinki, Finland), where the ground points were automatically classified and manually edited when needed.

The forest plots were revisited in December 2017 to update the field reference. Possible changes since 2014 were first visually interpreted from the ULS data with respect to the existing reference tree maps. Among the 24 plots, 22 plots remained almost unchanged. One plot was completely cut. For another plot, the ULS data mis-matched the plot location. Therefore, 22 plots were employed in this study. In the 22 plots, altogether 72 trees had been felled in 18 plots during 2014-2017. The maximum number of felled trees in a sample plot was 10 , which accounted for only a small proportion of the plot tree population. Thus, the classification of complexity categories did not change for the sample plots. The results of the ULS evaluations are therefore comparable with the results from TLS and MLS/PLS previously reported in other studies (i.e., Liang et al. 2018a, 2018b).

\section{In situ measurements using TLS and MLS/PLS}

TLS data were collected in 2014 using Leica HDS6100 (Leica Geosystems AG, Heerbrugg, Switzerland) with a multi-scan approach, that is, one scan at the plot center and four scans at the four quadrant directions. No pre-scan preparations were implemented in the field measurements. The data were registered using artificial spheres. The mutual scan registration accuracy was at a $2-\mathrm{mm}$ level. The point spacing was $15.7 \mathrm{~mm}$ at a $25-\mathrm{m}$ distance to the scanner in both horizontal and vertical directions.

The kinematic in situ measurements, namely, MLS from an all-terrain vehicle and PLS from a backpack, were also collected in 2014 (Liang et al. 2018b). The core measuring system for both platforms was identical, namely, AkhkaR2 (Finnish Geospatial Research Institute, FGI, Finland). Both platforms used the same scanning parameters: scanning frequency of $95 \mathrm{~Hz}$, which resulted in an approximate 4- $\mathrm{cm}$ on ground point spacing along the profile at a range of $35 \mathrm{~m}$ and an on ground profile spacing of $1.0-1.4 \mathrm{~cm}$ at a typical platform moving speed of $1.0-1.45 \mathrm{~m} / \mathrm{s}$.

\section{ULS measurements}

The ULS data were collected in September 2017 using a Riegl RiCOPTER with VUX-1UAV (RIEGL, Horn, Lower Austria, Austria), as shown in Fig. 3. The UAV campaign lasted for 3 days and the average time spent on UAV flight per plot was $10-20 \mathrm{~min}$. The flight altitude was 


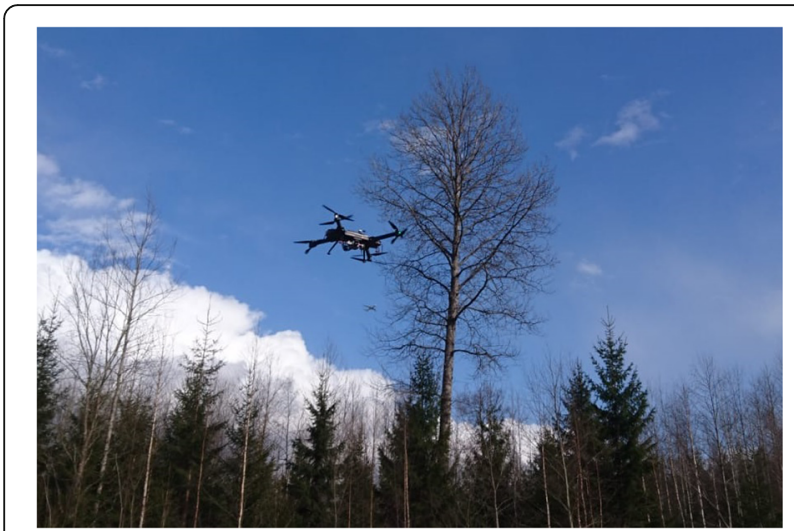

Fig. 3 The RiCOPTER ULS platform. (Image Courtesy: Timo Toivonen)

approximately $50 \mathrm{~m}$ above the ground. Each plot was covered by $4-5$ flight lines. The overlap of all flight lines at the plot area was typically high, e.g., $100 \%$.

The GNSS-IMU system installed on the UAV was Applanix AP20 GNSS-Inertial System (Trimble Applanix, Ontario, Canada). The position measurement accuracy was better than 0.1 and $0.2 \mathrm{~m}$ in horizontal and vertical directions, respectively. The roll, pitch and heading were measured at an accuracy of better than 0.015, 0.015 and 0.035 degrees, respectively. These give a point location accuracy of better than $3.6 \mathrm{~cm}$ at the nadir and $7.1 \mathrm{~cm}$ at the far end of the field-of-view at $50 \mathrm{~m}$ above the ground, if the positioning and ranging errors are left out of consideration.

The applied laser sensor was a Riegl VUX-1UAV. The scanner was mounted to scan nadir profiles and it operated at the $1550-\mathrm{nm}$ wavelength. The maximum measurement range of the scanner was $300 \mathrm{~m}$. The beam divergence was $0.5 \mathrm{mrad}$, providing $2.5-\mathrm{cm}$ and $5.0-\mathrm{cm}$ footprints at $50-\mathrm{m}$ and $100-\mathrm{m}$ distances from the scanner, respectively. Since the targeted flight altitude was $50 \mathrm{~m}$, the $50-\mathrm{m}$ and $100-\mathrm{m}$ distances corresponded to the scanning distances at nadir and at far end of the field of view. In order to gain better stem visibility, the data were collected with a 120-degrees field-of-view and a $550-\mathrm{kHz}$ laser pulse rate, resulting in 106 scan lines per second and in a 0.07 -degrees (1.2-mrad) measurement resolution along each scan line. Alone each scan line, the on ground point spacing was $6.1 \mathrm{~cm}$ at nadir and $24.4 \mathrm{~cm}$ at the far end of the scan line. The typical flight speed was $2.0-4.0 \mathrm{~m} \cdot \mathrm{s}^{-1}$, resulting in a $2.0-4.0 \mathrm{~cm}$ on ground spacing between scan lines. The point density was around 100 800 points $\mathrm{m}^{-2}$ on the horizontal ground surface if only one echo per pulse was considered. In practice, due to the high overlaps between flight lines, the point density increased vastly, resulting in $4000-18,000$ points $\cdot \mathrm{m}^{-2}$ at the sample plot areas. Two Sony ILCE-6000 cameras were also mounted on the UAV for colorizing the point cloud. Figure 4 illustrates the examples of colored ULS point cloud data in the three stand complexity categories. Color information was not used in the following processing.

The visibility of trees varied significantly according to tree species and forest structure, as shown in Fig. 5. For example, pine trees in easy forest stands typically had an excellent visibility and the stem was visible for most of its length. For spruce trees, the visibility of the stem decreased significantly as the tree stem can be either partly visible or totally occluded by the tree's own canopy and/ or by canopies of the surrounding trees.

Figure 6 illustrates a pine tree under moderate occlusion in the ULS data. ULS points at five different stem heights are shown in the subfigures. The stem structure is visually identifiable in two lower slices, but is totally missing in other three upper slices, indicating the impact of tree's own crown on its stem digitization.

Geometric inconsistency among different flight lines was visible in the delivered point clouds. Possible reasons for such errors include registration errors, influences of wind, and/or varying geometric accuracies from different flight lines at identical locations. Figure 7 shows four examples of mismatches between the flight lines, which

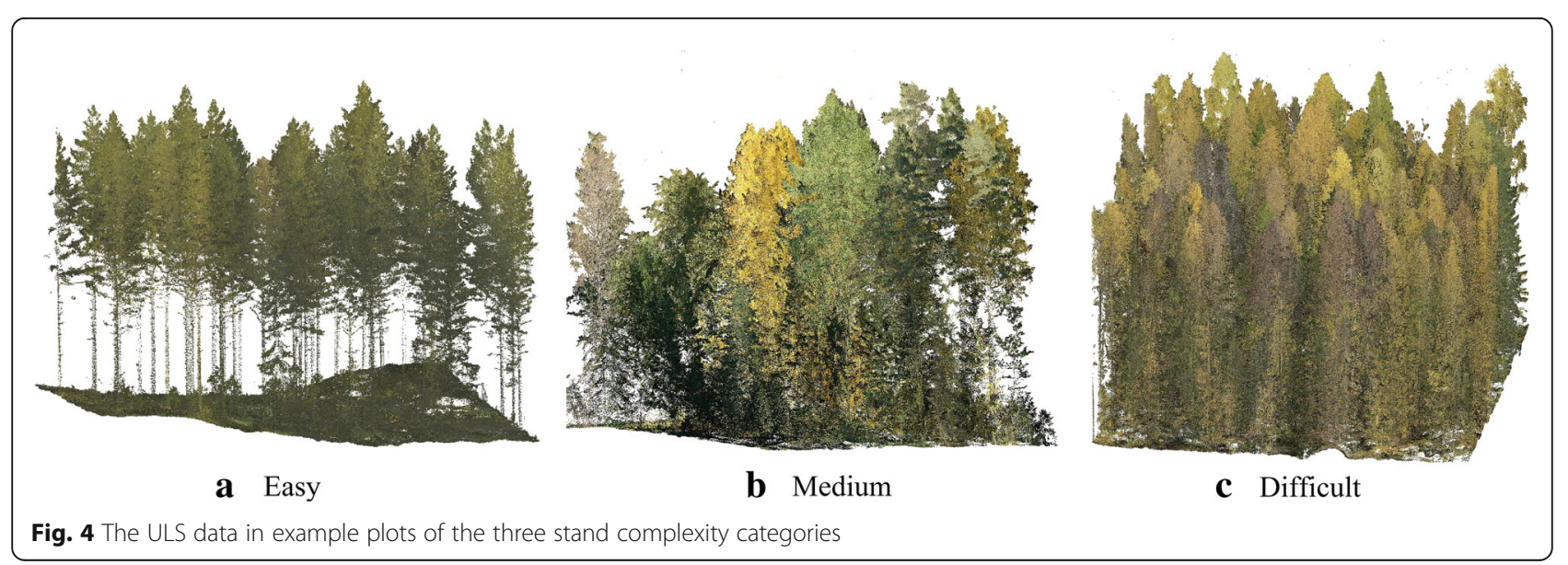




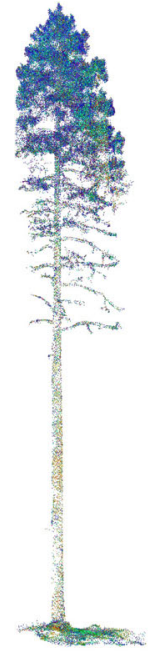

a
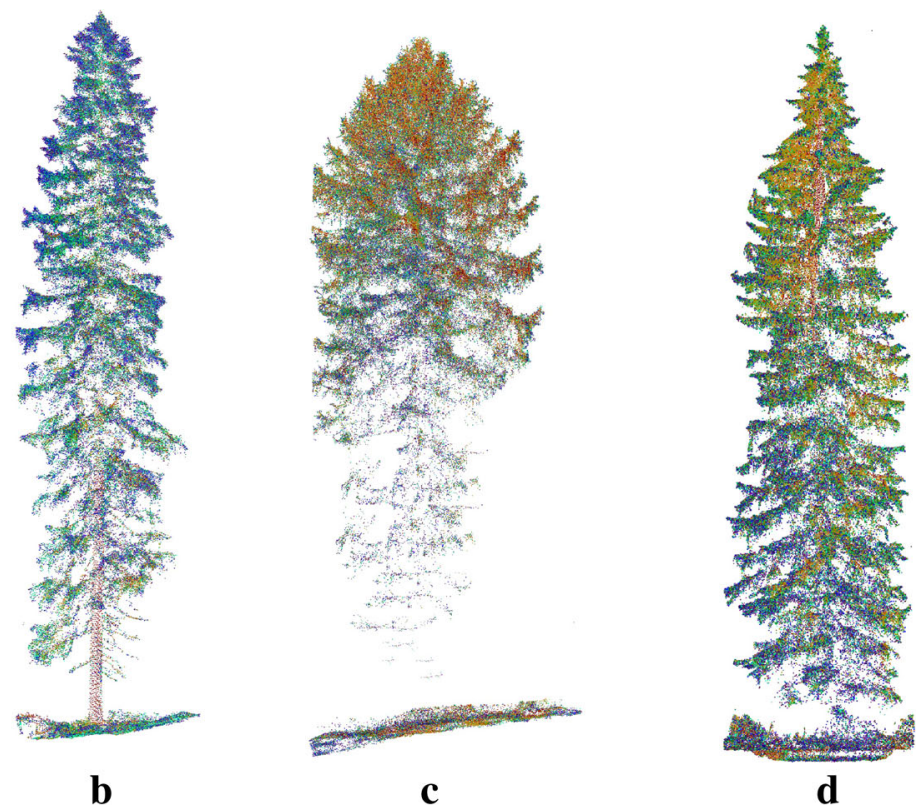

Fig. 5 Examples of trees captured in the ULS point clouds. a) A Scots pine tree standing in an easy plot; the stem is visible for the most of its length. b) A Norway spruce from an easy plot, lower part of the stem is well visible, while the foliage occludes the upper part of the stem. c) A Norway spruce from a difficult plot, the tree's own crown, surrounding canopies and understory occlude the entire stem. d) A Norway spruce from a medium plot. The upper part of the stem is identifiable, while lower part of the stem is mostly occluded

indicate the challenge of tree parameter estimation brought by geometric inconsistencies.

\section{Automated retrieval of tree and plot parameters}

The point cloud from stationary TLS, MLS/PLS and ULS, were processed through the same processing chain as described in Liang et al. (2018b). The specialty here is that the ULS data were understood equal to the terrestrial point cloud and processed with a stem detection and modeling algorithm that was identical to the other terrestrial point clouds.

In a preprocessing stage, the original point cloud was first sampled through an equivalent sampling method. The point cloud was digitized into a voxel space, and in each voxel the point closest to the center of gravity was selected as the representative point for the point distribution within the voxel. The voxel size was $1 \mathrm{~cm}$ for both the TLS and MLS and $0.5 \mathrm{~cm}$ for the ULS considering the given density of raw point clouds. The DTM was reconstructed using a morphological filter and linear interpolation. The point cloud was firstly rasterized in 2D. The lowest point in each pixel $(20 \mathrm{~cm}$ by $20 \mathrm{~cm})$ of a $2 \mathrm{D}$ raster space was selected as a seed point and the largest connected group was interpreted to be part of the ground. Detached groups were accepted as ground if they were smoothly connected with the accepted ground. Due to the above canopy viewing position, ULS collects tremendous amount of canopy points that are less important for the stem analysis. Therefore, a canopy filtering was applied to remove the topmost canopy layer (i.e., 20\% topmost) before the stem detection and modeling. After the stem detection, all canopy points were used in the tree height estimation.

Stem points were identified through a point-based approach. Points on stems were identified by analyzing the structure in their immediate neighborhood using principal component analysis. Tree stem models were built from the recognized stem points as a series of $3 \mathrm{D}$ cylinders representing the changes in the growth direction of stems. The $\mathrm{DBH}$ and location of a stem were then estimated from the cylinder element at breast height (1.3 $\mathrm{m}$ above the ground). The stem curve was estimated from the cylinder elements at predefined heights. The tree height was estimated differently for big and small trees, which were separated according to a $\mathrm{DBH}$ threshold of $15 \mathrm{~cm}$. Big trees were assumed mostly to be dominant or co-dominant trees that are exposed directly to the sunlight and no trees are above them (Wang et al. 2016). The tree top was assumed to be the highest point around the stem. The small trees were mostly intermediate and suppressed trees, and the treetop was therefore found from the largest connected point group around the tree stem. For both big and small trees, the elevation difference between the tree top and the DTM beneath it was used as a height estimate for the tree.

The MLS/PLS data were processed with one additional step. Since a tree may be observed several times from 


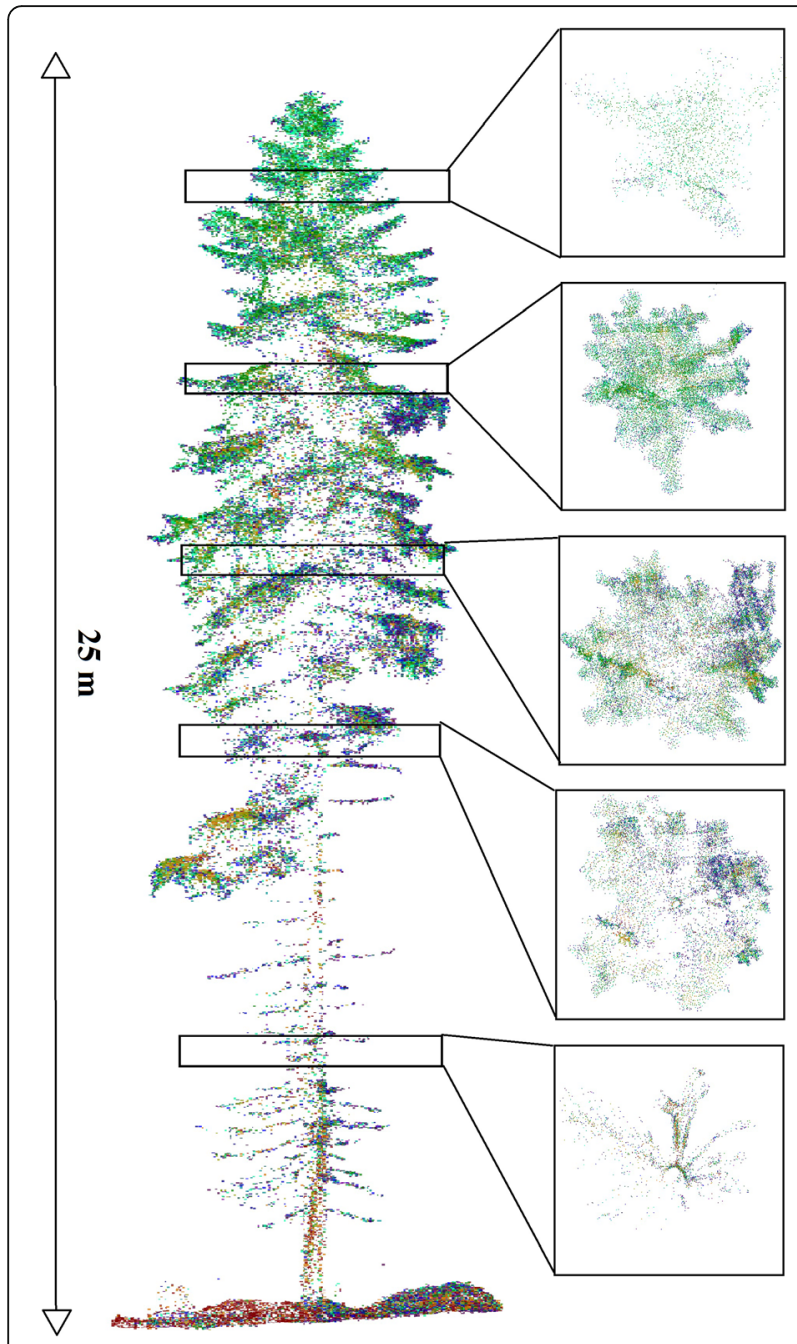

Fig. 6 The point cloud coverage of a Scots pine tree in a medium forest plot in the ULS data, and its horizontal profiles at five different heights above the ground level. Different colors show the point's origin from different flight lines. The figure illustrates impacts of tree crown on the stem digitization

different trajectories and spatial inconsistency from different observations prevails in the mobile data, the tree mapping followed the multi-single-scan type of processing (Liang et al. 2018a).

\section{Retrieval of tree and plot parameters from ULS data by manual measurements}

In addition to the automated processing, 7 plots, 3 from easy, 2 from medium and 2 from difficult categories, were randomly selected and manually measured in order to benchmark a data-level accuracy, thus, to enhance understandings of the automatically derived tree parameter estimates. The manually measured tree parameters include the tree position, the $\mathrm{DBH}$ and the tree height of individual trees that are visually identifiable from the ULS data. A circle at the breast height was fitted to each visually identified tree. If only few or none points were recorded for a stem at the $\mathrm{DBH}$ height, a circle at a higher position was searched for and fitted when possible. The tree position and the DBH were therefore the center of the fitted circle and the diameter of the fitted circle, respectively. The treetop and the stump were also visually identified, and the elevation difference between the tree stump and top was recorded as the tree height.

\section{Evaluating the accuracy of individual tree parameters}

The performance of TLS, MLS/PLS, ULS was evaluated by comparing the accuracies of the estimated tree-level parameters with respect to the field measurements.

The evaluation followed the procedure in Liang et al. (2018a). The detected trees were matched with the reference trees based on the horizontal stem locations and the DBHs of the trees. The search distance was $50 \mathrm{~cm}$ for TLS and ULS, and $150 \mathrm{~cm}$ for the MLS/PLS. The mapping accuracy was evaluated using the completeness, which indicates the proportion of reference trees that were automatically detected. The accuracy of the tree position, tree height, DBH, stem curve, stem volume and AGB were all evaluated using the relative Root Mean Squared Error (RMSE) and relative bias, both in percentages, with an exception of the tree location where only the absolute RMSE was calculated. The evaluation was carried out at an individual-tree-level, i.e., the estimated and reference parameters were compared, and reported at a plot-level, i.e., relative RMSE and bias were calculated at a plot level and further averaged at the plot-complexity-category-level. The results are reported separately for each stand complexity category.

\section{Results}

The feasibility of the forest in situ measurements from the aerial perspective was investigated through the accuracy of parameter estimates and also through a comparison among the performances of currently available terrestrial and areal data sources, i.e., the ULS, multi-scan TLS and MLS/PLS.

\section{Stem mapping}

The stem mapping results from ULS, MLS/PLS and TLS are reported in Fig. 8. Similar to the MLS/PLS and TLS, ULS had a steady declining trend in the completeness of stem mapping when the stand conditions became more complicated. The manual stem mapping from ULS provided very similar results as the automated stem mapping from MLS/PLS and TLS. However, when processed with the automated algorithm, the completeness of ULS-based stem mapping was remarkably lower (approximately 50\% lower) than that of the MLS/PLS- and TLS-based results. Moreover, the decrease of ULS-based automated stem 

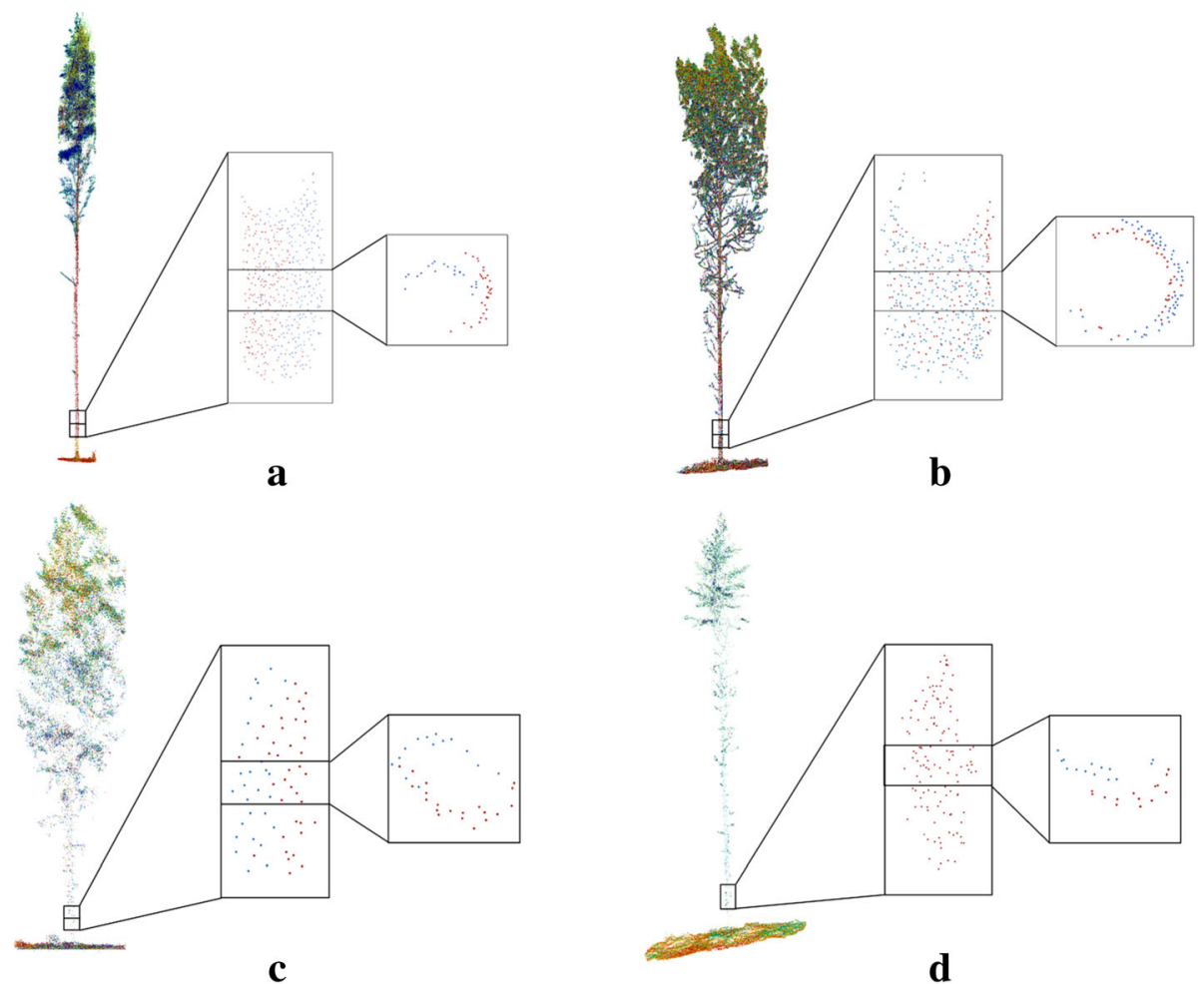

Fig. 7 An illustration of geometric inconsistencies among flight lines in ULS point clouds. a) A Silver birch from an easy plot, with 15-cm DBH. b) A Scots pine from an easy plot, with $23-\mathrm{cm}$ DBH. c) A Scots pine from a difficult plot with $13-\mathrm{cm} D B H$. d) A Scots pine from a difficult plot with a DBH of $15 \mathrm{~cm}$. The subfigures in middle zoom in to a one-meter-long section of the stems at $+/-50 \mathrm{~cm}$ of the breast-height at $1.3 \mathrm{~m}$; the subfigures to the left show the horizontal profiles of 20 - $\mathrm{cm}$-thick slices of the stems at $+/-10 \mathrm{~cm}$ of the breast-height. The different colors (red/blue) show the flight lines of the points

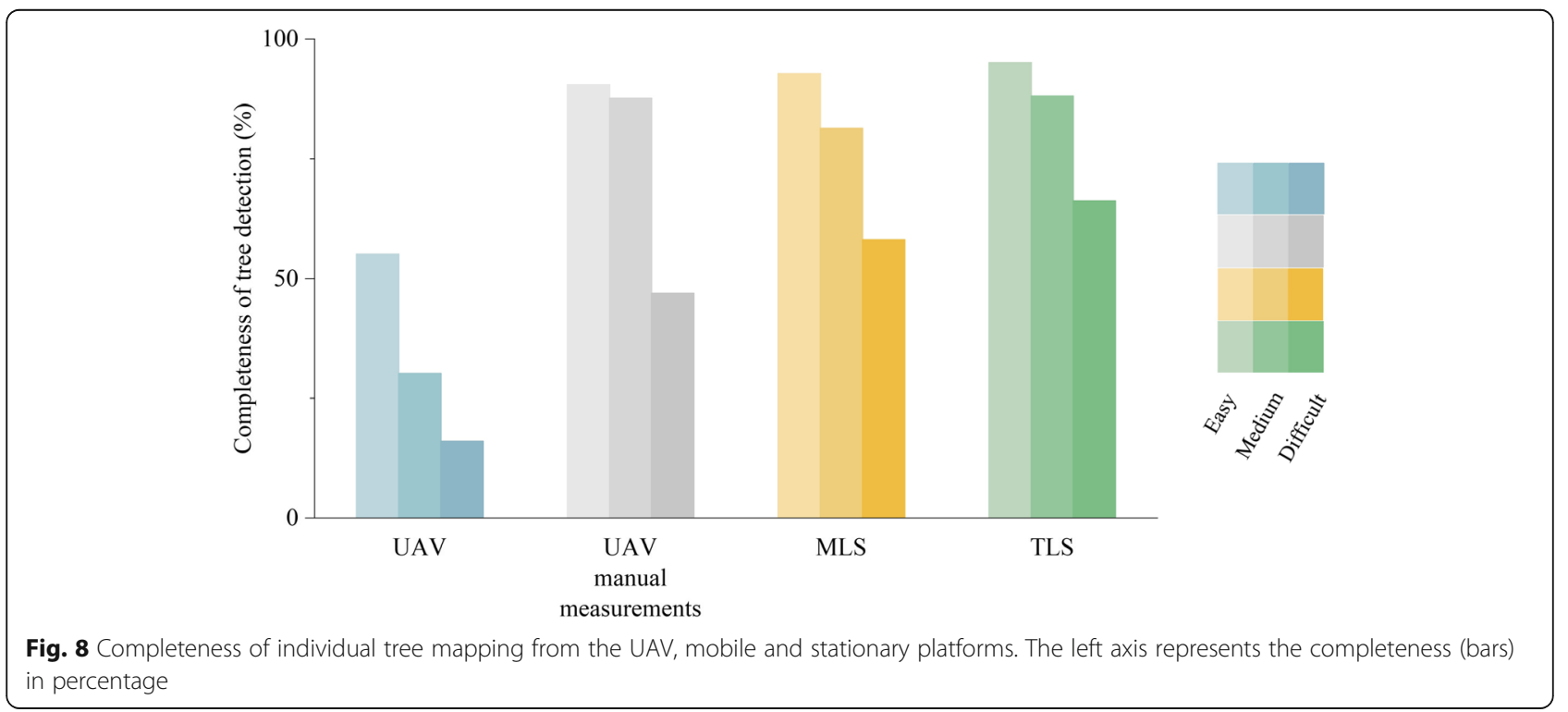


mapping completeness with respect to the increase of the stand complexity was more significant than that of the MLS/PLS- and TLS- based results.

These results suggest that, firstly, the state-of-the-art ULS data are capable to digitize similar amount of stems in forest plots as the other terrestrial point clouds; secondly, the stand complexity has more significant influence to the ULS-based stem digitization than to the other terrestrial point clouds. Even though the stem point cloud in ULS data (e.g., the density and the geometrical accuracy) is sufficient for human recognition, it is not yet comparable with the other terrestrial point clouds to support the automated stem detection, at least with the applied algorithm.

\section{Stem position}

The stem positioning accuracy from ULS, MLS/PLS and TLS are reported in Fig. 9. In general, the average RMSE of stem locations for digitized stems in ULS data was between $6.0 \mathrm{~cm}$ and $15.0 \mathrm{~cm}$ considering the stand complexity. The ULS-based stem positioning error is about two times as much as that of TLS-based results as shown in the Fig. 9. Compared to ULS, the MLS-based stem positioning accuracy was significantly lower due to the geometric inconsistency brought by the unstable GNSS signals under canopies.

\section{Diameter at breast height}

The relative RMSE (RMSE\%) and the relative bias (bias\%) of the DBH estimates from the three platforms are reported in Fig. 10.

The columns of "UAV" and "UAV (gross errors removed)" in Fig. 10 represent the evaluation results of the automated DBH estimates from ULS data. The differences between these two results were caused by gross errors, which mainly occurred at the stems of small trees that were digitized with low quality in the ULS point clouds. That is, the stems were digitized in the ULS data to an extent that is not enough for a reliable delineation by the automated algorithm. The stem modeling from ULS data produced large errors, for example, due to the low density, completeness, and geometric accuracy of stem points. Errors larger than $20 \mathrm{~cm}$ with respect to the reference were considered as gross errors. Gross errors accounted for only a small proportion of detected stems, that is, maximum three in a plot. Once the gross errors were removed, the RMSE\% of automated ULS-based DBH estimates became comparable to that of the manual recognition from ULS data, as well as to that of the automated DBH estimates from TLS and MLS/PLS data.

The RMSE\% of ULS-automated, ULS-manual, and automated estimates from MLS were at a same level $(15 \%-30 \%$ in different stand complexities), and they were twice as much as the RMSE\% of the TLS-based estimates. The bias\% of ULS and MLS suggested that both aerial and terrestrial mobile platforms lead to overestimated DBH. The overestimation from ULS is more significant than that from MLS, indicating the geometric accuracy at the stems is higher in the MLS compared to the ULS point clouds.

\section{The tree height}

The advantage of an aerial observation perspective for tree height measurements is clearly shown in Fig. 11. ULS presented the best performances across different stands among all point clouds due to a better visibility of the upper parts of crowns. On the other hand, the tree height is the only parameter, for which the ULS-based estimates outperformed the MLS- and TLS-based estimates.

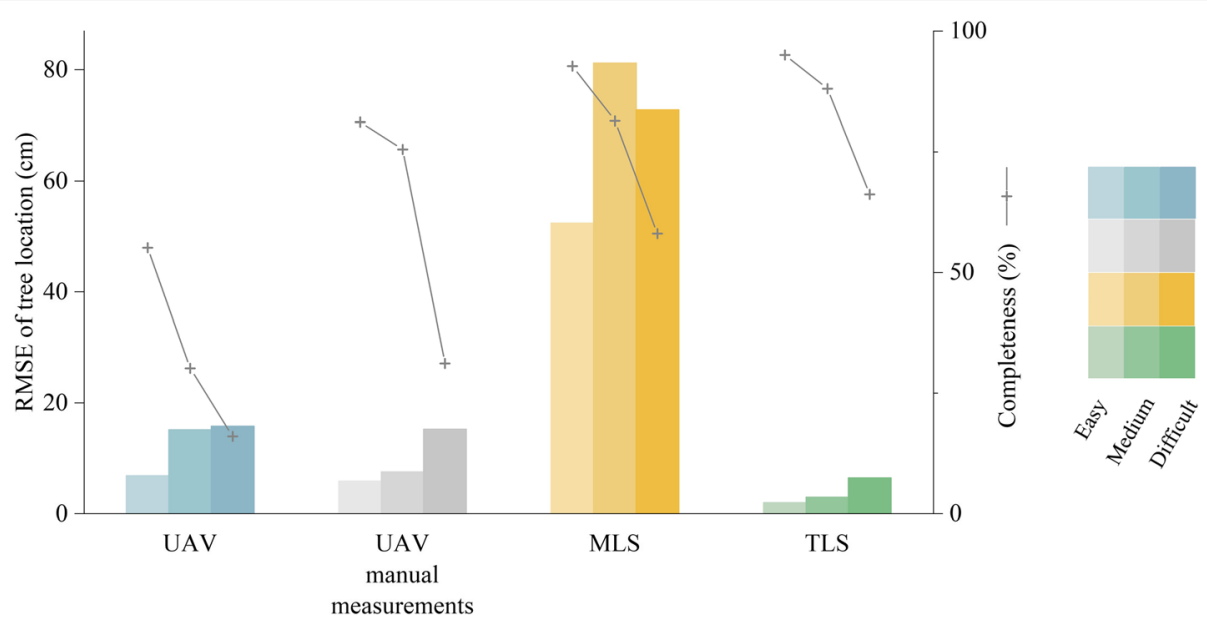

Fig. 9 Stem location estimation at the breast height from the UAV, mobile and stationary platforms. The left axis represents the RMSE (bars) in $\mathrm{cm}$ and the right axis represents the completeness (solid line with ' + ' markers) in percentages. In the completeness of manual measurements from the ULS data, only those trees for which the stem was identifiable at the breast height in the point cloud were taken into account 

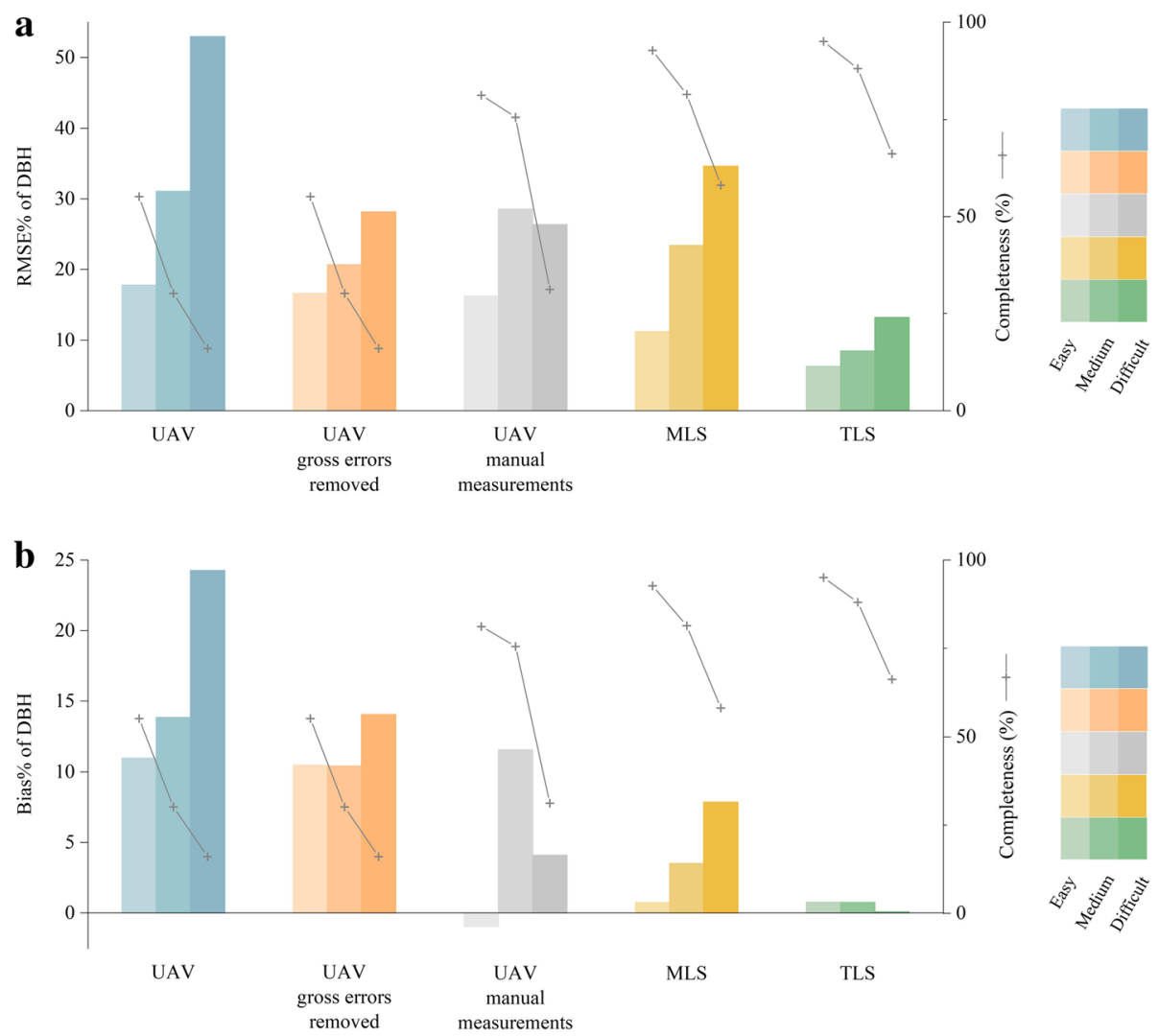

Fig. 10 Relative RMSE (a) and bias (b) of the DBH estimation from the UAV, mobile and stationary platforms. The left axis represents the RMSE and Bias values (bars), and the right axis represents the completeness value (solid line with ' + ' markers). All units are in percentages

The automated tree height estimates from ULS performed similarly as the manual mensuration in easy plots and better in medium and difficult plots. This however does not mean that the automated algorithm outperforms the manual identification in the treetop recognition. The difference is due to the amount of recognized trees, which is smaller in the automatic detection. Automatic algorithm detected only trees whose trunk was clearly visible in the data. This underlines the claim in the TLS benchmarking (Liang et al. 2018a) that the accuracy of individual tree parameter estimates should be analyzed together with the detection rate (i.e., completeness). Treetop identification in point clouds is a challenging task even for human eyes especially in medium and difficult stands where species are mixed and tree crowns are entangled with each other. Thus, treetops may be misinterpreted, leading to a larger error than that of the automated recognition where only the trees clearly recorded were measured. These results also indicate that the manual and the automated interpretation of point clouds perform very similarly in treetop recognition for trees that are clearly digitized.

\section{The stem curve}

The evaluation of stem curve estimates are reported in Fig. 12. While the RMSE\% of the stem curve estimates from ULS was similar to that of the DBH, the bias\% was almost doubled. This was caused by more gross errors and less accurate estimates due to, e.g., occlusions in the data. Similar to DBH and tree height estimates, the RMSE\% and bias\% increased along the increased stand complexity.

The percentage of tree height covered (PHC, the extracted stem length covered by the stem curve divided by the tree height) of ULS-based stem curve estimates was in general higher than that of MLS and lower than that of TLS. The PHC value of the stem curve estimates based on ULS was expected to be higher than that of the terrestrial point clouds from TLS and MLS, because ULS has a favorable viewing position to digitize upper part of tree stems. However, the results suggest that the stem curve modeling from ULS can be disturbed by the missing parts in the middle of the stems, therefore, the clearly digitized upper stem parts can hardly be included in the stem curve model. While outperforming the MLS, ULS still cannot 


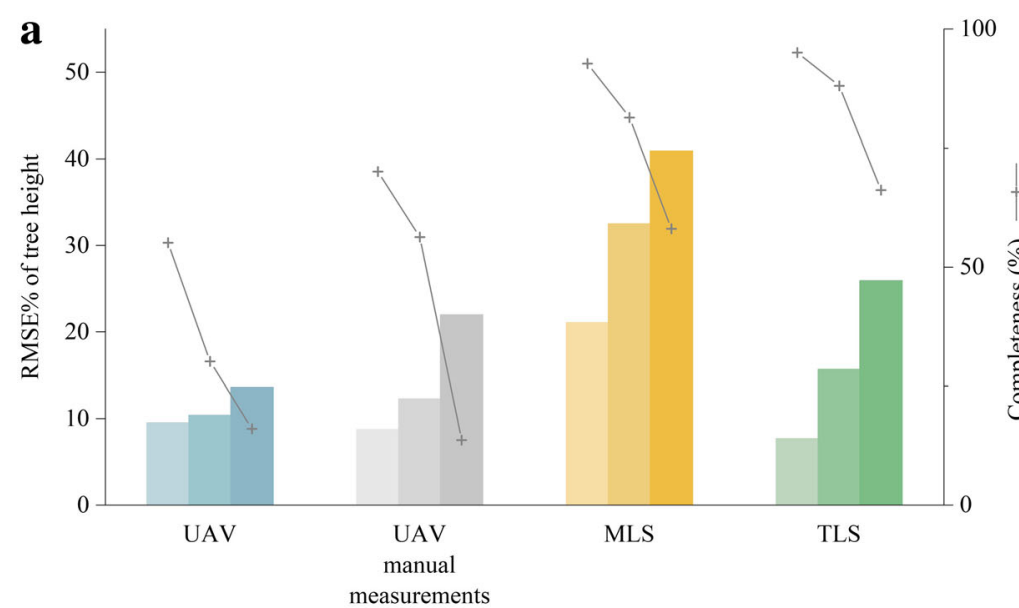

00
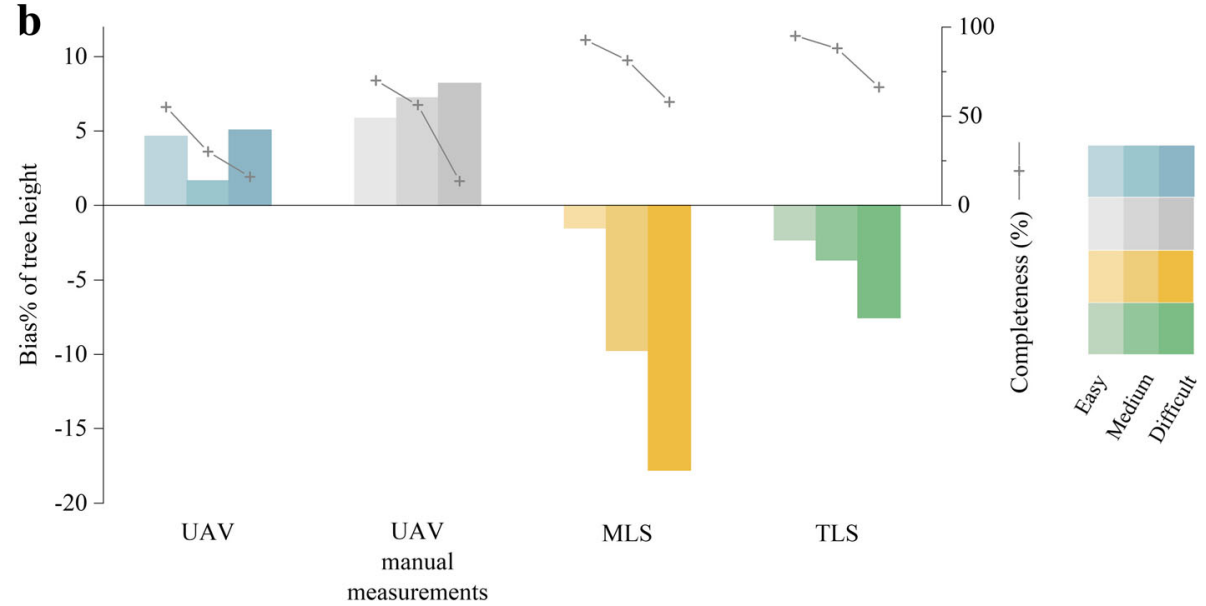

Fig. 11 Relative RMSE (a) and bias (b) of the tree height estimates from the UAV, mobile and stationary platforms. The left axes represents the RMSE\% and bias\% (bars), and the right axes represents the completeness (solid line with ' + ' markers). Tree heights are retrieved from ULS both automatically and manually. In the completeness of manual measurements, only trees with an identifiable tree top in the point cloud were taken into account. All units are in percentages

compete with TLS in the stem curve modeling in the sense of completeness, i.e., PHC. More discussion about stem curve estimates is given in the Section of Discussion.

\section{Stem volume and total tree biomass}

The results of the stem volume estimates are reported in Fig. 13. The stem volume is a function of stem curve, but it is proportional to the square of the radius of the stem. Therefore, the increase of ULS-based volume error with respect to the stand complexity is higher compared to the increase of ULS-based stem curve error (Fig. 12).

The results of the total tree biomass estimation are reported in Fig. 14. The RMSE\% values in easy plots increases from $11.1 \%$ in TLS to $24.6 \%$ in MLS and to $37.6 \%$ in ULS. In the medium and difficult plots, the differences between different systems become larger. Since the stem curve and DBH accuracies play a more significant role in the volume and biomass estimates (Liang et al. 2018a), the more accurate tree height estimates of the ULS do not improve the ULS-based stem volume and total tree biomass estimates observably.

\section{Discussion}

ULS has unique properties that may change the forest field inventory profoundly. It outperforms other terrestrial remote sensing systems in the data collection speed since the platform movement is totally free from ground obstacles. So far, the geometrical accuracy of the ULS point clouds has not yet reached the level of the TLS point clouds, which is required in practical applications. However, as the sensors and the platforms evolve constantly, the ULS-based forest in situ observations deserves more studies. 

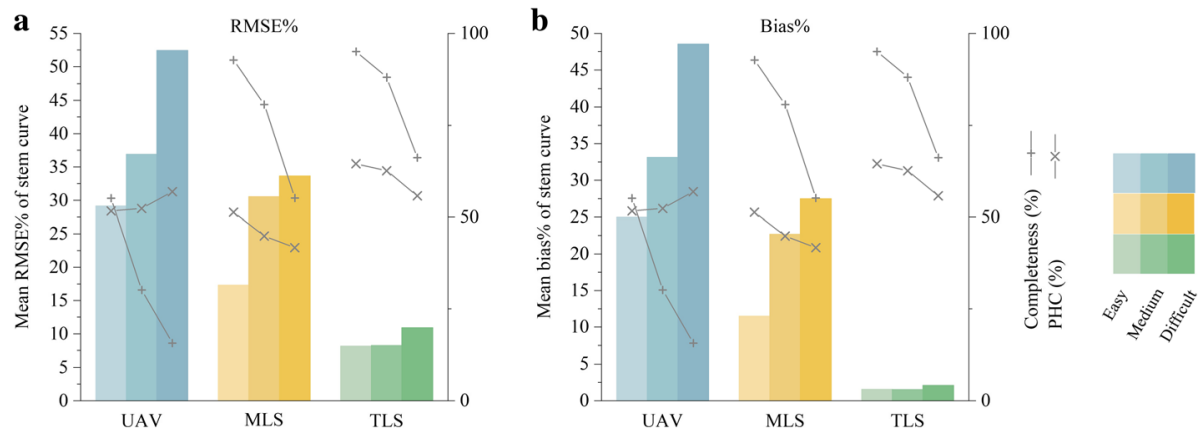

Fig. 12 Relative RMSE (a) and bias (b) of the stem curve estimates from the UAV, mobile and stationary platforms. The left axes represent the RMSE\% and bias\% values (bars). The right axes represent the completeness (solid line with ' + ' markers) and percentage of tree height covered (PHC, solid line with ' $x$ ' markers). All units are in percentages

\section{Pros and cons of the above-canopy view perspective in forest field inventories}

The main motivation of applying mobile platforms (e.g., MLS/PLS/ULS) in forest inventories is three-fold. Firstly, mobile platforms improve the efficiency of data collection significantly. Secondly, the area of interest is more thoroughly covered by the kinematic data collection compared to stationary scans. Thirdly, the occlusion effects can be mitigated by moving the observation viewpoint around the targets.

Uplifting the viewpoint from the ground to the air using a UAV platform indeed raises the mobility to a level that has never been seen before. Due to the absence of obstacles along the path, ULS's mobility is even higher than what MLS can provide. In comparison with TLS and MLS, the forest plots are covered more comprehensively in the ULS data, that is, close to $100 \%$ plot coverage in each and all flight lines. However, currently, such coverage improvement is present mainly in the horizontal dimension. In the vertical dimension, ULS is able to digitize trees comprehensively if the viewing geometry is ideal. Complete coverage of a tree from the stump to the top can be achieved if occlusions, e.g., from the canopies and other nearby trees, are absent, e.g., for an isolated tree. However, occlusions still exists which remarkably reduce the ULS data coverage of individual trees in the vertical direction, specifically at the lower or middle parts of the stems. As such, recent application of UAV platform does not yet reach the objective of mitigating the occlusion effects present in terrestrial technologies. Results in this study suggest that the mobile platforms on the ground lessen the occlusion much more successfully, even though the ground mobile platforms suffer from the geometric inconsistency due to GNSS signal losses.

Unlike the terrestrial point clouds where the occlusion effects are mainly caused by the bushes, small trees and lower parts of trees, in the ULS point clouds, the occlusions are caused significantly by the upper parts of the canopies. Data collection in leave-off season might be able to mitigate certain occlusions of deciduous trees, but cannot solve the problems in coniferous forests. As illustrated in Figs. 6 and 7, tree stems may be fully, partially or barely

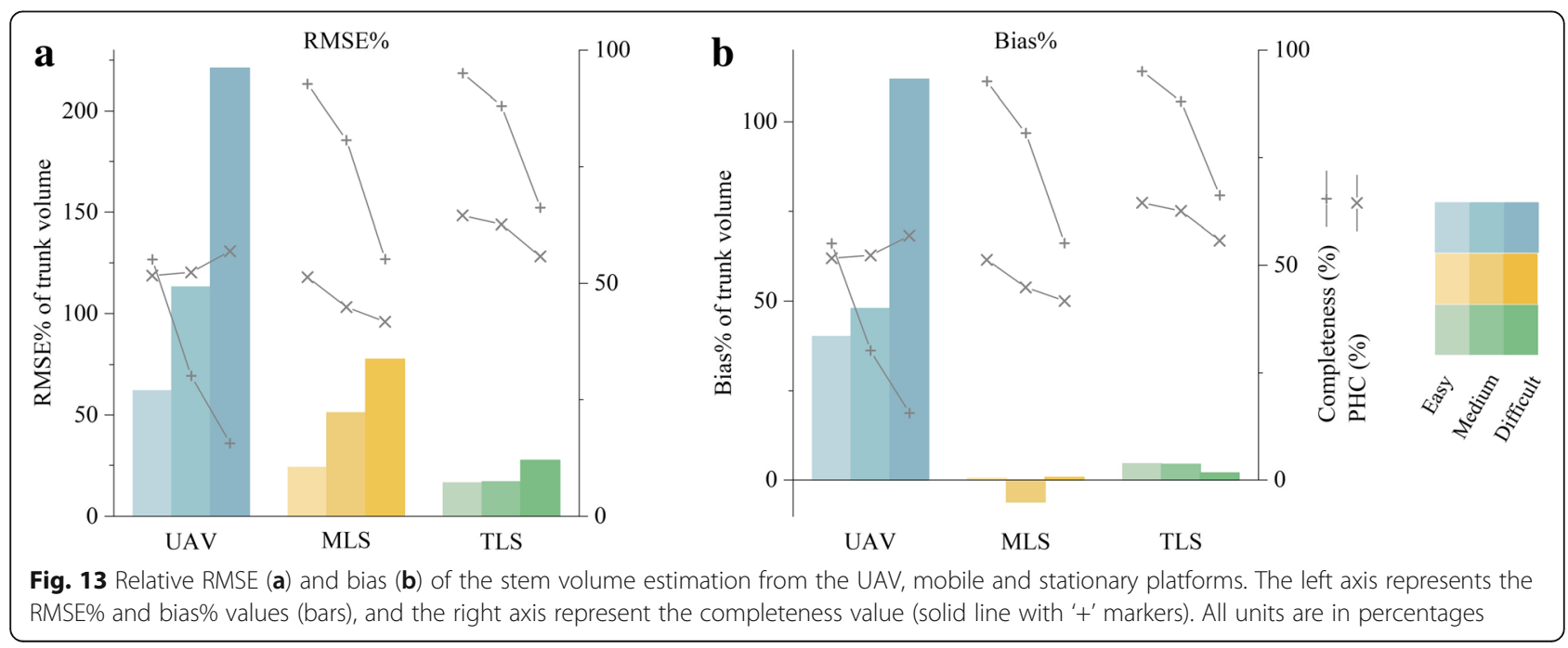



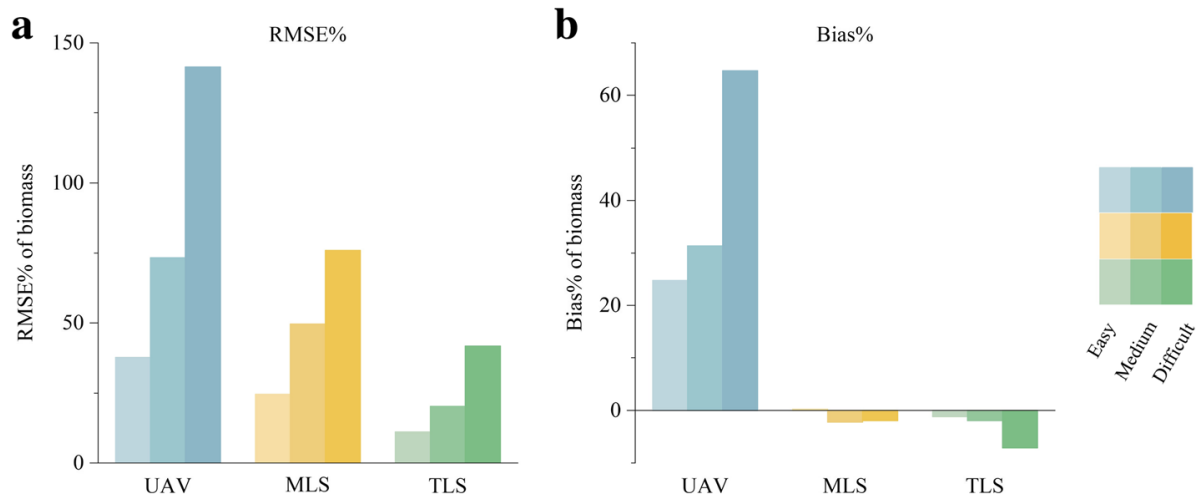

Fig. 14 Relative RMSE (a) and bias (b) of the total tree biomass estimation from the mobile and stationary platforms. The left axis represents the RMSE\% and bias\% values (bars), and the right axis represent the completeness value (solid line with ' + ' markers). All units are in percentages

digitized by ULS, depending on the scanning geometry, species and forest stand conditions. The applied ULS system in this study is a high-end product. The point clouds from the survey-grade laser scanner and the fairly good navigation system represent a high quality point cloud data in the ULS domain in terms of the geometric accuracy and point density. With the applied high-end ULS, the capability of stem digitization from above canopies seems to be, however, still limited.

\section{Data acquisition configurations}

The density and geometric consistency of the point cloud data from ULS are influenced by the forest stand conditions, e.g., the structure and species of the forest, and by the data acquisition configurations, e.g., the setups of the scanner, positing system and flight lines.

In general, the point density of a scanner with the rotating mirror scanning mechanism is a function of the Pulse Repetition Rate (PRR), the Field of View (FOV) and the flight height and speed, which determines the point spacing within and between the scan line(s). A high PPR, low flight height, slow flight speed and small FOV lead to a high point density, and vice versa. Also, high overlap rate between flight lines over the forest site increases the point density. Higher point density typically achieve better canopy penetration capacity. But this is not guaranteed. Forest conditions have significant impacts on the point penetration capability.

Using the sensor and platform configurations applied in this study as described in the Section of ULS measurements, the on ground point spacing of the ULS point cloud is $6-15 \mathrm{~cm}$ within a scan line and $2-4 \mathrm{~cm}$ between the scan lines, which produced 4000-18,000 points. $\mathrm{m}^{-2}$ point density. However, such data are still not enough to record trees comprehensively, as shown in Figs. 5 and 6. In addition, the stems of small trees (e.g., $5-10 \mathrm{~cm} \mathrm{DBH}$ ) are recorded vaguely in the ULS point cloud, taking into account the occlusion effects under forest canopies.

Multiple flight lines further increase the point density, and consequently increase point coverage on the targets. However, the geometrical consistency inside the point cloud may decrease because of the inaccuracy of the registration between flight lines, as shown in Fig. 7, which may dwarf the benefit of multiple flight lines.

Generally speaking, the best practice of the ULS measurement, e.g., the FoV, flight height, speed and overlap rate between flight trajectories, are not yet established. These research questions deserve more research in future.

\section{Point geometrical accuracy matters}

The geometrical accuracy of a point in a mobile point cloud from a linear laser scanner is determined by the scanner settings, such as the beam divergence and FoV, and platform status, such as rotations and sensor-target distances.

In the applied ULS system, the angle measurement accuracy of the platform is better than 0.015 and 0.035 degrees for roll/pitch and heading, respectively. If the GNSS positioning and laser ranging errors are not considered, this angle measurement accuracy means that, from a $50-\mathrm{m}$ altitude, a laser point's position accuracy of a laser point on the ground is better than 3.6 and $7.1 \mathrm{~cm}$, at the nadir and at the far end of the field-of-view, respectively. Such data accuracy is insufficient considering the required $1-2 \mathrm{~cm}$ accuracy of DBH estimates in NFIs.

In addition, the survey-grade laser scanner applied in the ULS system has a $0.5 \mathrm{mrad}$ laser beam divergence, which corresponds to a $2.5-\mathrm{cm}$ footprint at nadir and a 5 -cm footprint at the far end of FOV for a 50-m flight altitute. While negligible in TLS data due to the generally smaller beam divergence (e.g., $0.2 \mathrm{mrad}$ ) and the shorter scanner to target distances in forests (e.g. typically under $40 \mathrm{~m}$ ), the influence of the beam divergence should be taken into account when using ULS data since it 
negatively affects the positioning accuracy of the points in the point cloud.

Moreover, the geometrical accuracy of points is heterogeneous through out a point cloud, namely, higher accuracy at nadir and lower accuracy at far end of FOV, which brings further challenges on point cloud registration between different flight lines. The relatively large uncertainties in ULS- and MLS/PLS-based tree parameter estimates can thus be understood as a result of error propagation from the platform positioning and orientation, and the geometric accuracy of points from the laser sensors. A slightly better performance can be observed from MLS/ PLS than ULS, probably because MLS/PLS can achieve a similar performance as TLS in short periods of time. When the time span is short, the positioning drift is small and the relative positions between scanner and target are close to stable. Furthermore, the multi-single-scan principle for stem modeling applied in the MLS/PLS data processing shortens the time span and thus also reduces positioning errors that propagate to the point cloud data.

\section{Outlook of ULS for in situ measurements of forest}

ULS is the first aerial technique that achieves a stem digitization at a similar level of detail as TLS. Despite the recent technical challenges, the amount of recognizable stems in ULS data with human interpretation is similar to that with automated detection from TLS data.

So far, the geometrical accuracy of the ULS point clouds has not yet reached the level achieved through terrestrial LS systems. Even with the reasonably good UAV positioning system, the absolute 3D accuracy of a ULS point (3.6-7.1 cm on ground) is not yet accurate enough to estimate DBH and stem curve with a required accuracy. However, ULS outperforms terrestrial systems on the tree height estimations. Moreover, ULS provides a new in situ observation possibility that the observations are performed above the forest site. ULS frees the platform from any on-ground mobility constrains and enables fast and vast observations. Therefore, ULS may have a fundamental impact on the forest inventories.

The geometric inconsistencies is ubiquitous in point clouds, as results of winds, measurement inaccuracy and registration errors (Liang et al. 2018b; Pyörälä et al. 2018). While the wind impacts can hardly be eliminated, accuracy in measurement and registration can be improved. Smaller beam divergence, more accurate range measurement and higher angular resolution should provide higher measurement accuracy. Small FOV and slow flight speed can also reduce point spacing on the ground in ULS data.

All mobile systems in the air and on the ground face the same challenges of accurate registration between trajectories. The inconsistencies brought by overlapping trajectories in the ULS data appear less significant than that in the MLS/PLS point cloud if measurements from all trajectories are merged. The reason for this is that the GNSS signal degenerates under the forest canopies significantly, which hinders the accurate registration in terrestrial systems. In ULS, higher-quality GNSS-IMU system improves the positioning accuracy and visionaided positioning (e.g., from structure-from-motion) can improve the orientation accuracy (e.g., Suomalainen et al. 2014). Actually, the registration problem in ULS may be slightly easier to solve than that in terrestrial platforms since the initial location parameter from GNSS is fairly accurate.

Even though the completeness and the geometric accuracy of stem digitization in ULS data is not as high as in other terrestrial systems, it records treetops in high detail. This is shown by the accurate tree height estimates in this study, which confirms the conclusion in a previous study (Wang et al. 2019). This hints that ULS most probably measures other canopy features accurately as well. Tree height and canopy features with higher resolution than those from ALS may serve as effective explanatory variables in the estimation of forest biomass, age and productivity. Meanwhile, ULS is most probably a suitable option in particular applications. ULS's areal point of view has the potential to mitigate the occlusion effects within canopy, thus the structure information within canopies may be studied at a higher level of detail than what is presently achievable. Point clouds collected from terrestrial platforms typically have a limited spatial coverage of tree structures inside the canopy. Consequently, the performance of the quantitative structure delineation decreases in modelling the stems and branches above the live canopy base, even with high-resolution TLS point clouds (Liang et al. 2018a; Pyörälä et al. 2019). In addition, considering time and cost efficiency, ULS is also a very competitive candidate for the applications that emphasize tree count and location rather than tree parameter mensuration, such as the inventory of young plots.

\section{Conclusion}

Forest in situ measurements play a fundamental role in forest assessment and management. The in situ measurements are currently experiencing rapid and profound changes, driven by new sensors (e.g., single photon laser scanning), new platforms (e.g., UAV and terrestrial kinematic platforms), and other new advances such as sensor miniaturization, price dropping, and the developments in signal processing techniques. UAV-borne laser scanning (ULS) is one of the latest progresses in the field in the last decade, with which, for the first time, forest in situ observations that directly record the stem structures from an aerial perspective is enabled.

This study gives the first strict evaluation of ULS-based in situ observations in varying forest conditions. It also acts 
as a benchmarking of available active remote sensing techniques for forest in situ mensuration, considering the comparisons between ULS, MLS/PLS and TLS. The tree and plot parameters automatically retrieved from ULS data were evaluated using field reference and compared to those retrieved from other prominent techniques. The $\mathrm{DBH}$, tree height and tree position were also manually retrieved from the ULS point cloud to evaluate the ULS data accuracy. The results indicate that the current off-the-shelf ULS has an excellent tree height/tops measurement performance. Because of the limitations in the positioning and angular measurement, the geometrical accuracy of the ULS data, especially at the stem parts, does not yet reach the level of terrestrial point clouds collected using MLS/PLS and TLS. Nevertheless, the unbeatable high mobility and fast data acquisition makes the ULS a very attractive option in forest investigations, especially in applications emphasizing crown shape and tree mapping.

\section{Abbreviations}

3D: three dimensional; AGB: Above Ground Biomass; ALS: Airborne Laser Scanning; DBH: Diameter at the Breast Height; DTM: Digital Terrain Model; FOV: Field of View; GNSS: Global Navigation Satellite System; IMU: Internal Measurement Unit; LS: Laser Scanning; MLS: Mobile Laser Scanning; NFI: National Forest Inventory; PHC: Percentage of tree Height Covered; PLS: Personal Laser Scanning; PPR: Pulse Repetition Rate; RMSE: Root Mean Square Error; TLS: Terrestrial Laser Scanning; UAV: Unmanned Aerial Vehicle; ULS: UAV Laser Scanning

\section{Acknowledgements}

Not applicable.

\section{Funding}

Finnish Academy projects "Centre of Excellence in Laser Scanning Research (CoE-LaSR) (272195)", Competence Based Growth through Integrated Disruptive Technologies of 3D Digitalization, Robotics, Geospatial Information and Image Processing/Computing - Point Cloud Ecosystem (293389/314312), and the Business Finland DroneKnowledge project (1617/31/2016).

\section{Availability of data and materials}

Six plots of the applied Multi-scan TLS were published with open access for non-commercial uses.

\section{Authors' contributions}

Xinlian Liang, Yunsheng Wang designed the experiments; Xinlian Liang processed the data; Yunsheng Wang analyzed the results; Jiri Pyörälä carried out manual measurements in ULS data; Jiri Pyörälä, Matti Lehtomäki, and Xiaowei Yu participated the evaluation and figure preparation; Harri Kaartinen coordinated the ULS data collection; Antero Kukko developed the systems for PLS, MLS data collection; Aimad Issaoui, Jiri Pyörälä and Matti Lehtomäki carried out reference data verification; Eija Honkavaara, Olli Nevalainen, Matti Vaaja and Juho-Pekka Virtanen contributed to discussion of ULS geometric accuracies; Masato Katoh and Songqiu Deng contribute to the discussion of forest field inventory; Xinlian Liang and Yunsheng Wang coordinated the manuscript preparation; All authors contributed to the manuscript writing and editing.

\section{Ethics approval and consent to participate}

The subject has no ethic risk.

\section{Consent for publication}

All the data and relevant processing methods, including the automated generation of figures of evaluation results, belong to the host institution, namely, the Finnish Geospatial Research Institute.

\section{Competing interests}

No competing interests.

\section{Author details}

${ }^{1}$ Department of Remote Sensing and Photogrammetry, Finnish Geospatial Research Institute, 02431 Masala, Finland. 'Department of Built Environment, Aalto University, 02150 Espoo, Finland. ${ }^{3}$ Institute of Mountain Science,

Shinshu University, Nagano Pref 399-4598, Japan.

Received: 7 November 2018 Accepted: 28 February 2019

Published online: 15 April 2019

\section{References}

Bauwens S, Bartholomeus H, Calders K, Lejeune P (2016) Forest inventory with terrestrial LiDAR: a comparison of static and hand-held Mobile laser scanning. Forests 7:127. https://doi.org/10.3390/f7060127

Brede B, Lau A, Bartholomeus H, Kooistra L (2017) Comparing RIEGL RiCOPTER UAV LiDAR derived canopy height and DBH with terrestrial LiDAR. Sensors 17:2371. https://doi.org/10.3390/s17102371

Chisholm RA, Cui J, Lum SKY, Chen BM (2013) UAV LiDAR for below-canopy forest surveys. J Unmanned Vehicle Syst 01:61-68. https://doi.org/10.1139/ juvs-2013-0017

Dick AR, Kershaw JA, MacLean DA (2010) Spatial tree mapping using photography. Northern J Appl Forest 27:68-74

Forsman M, Börlin N, Holmgren J (2016a) Estimation of tree stem attributes using terrestrial photogrammetry with a camera rig. Forests 7:61. https://doi.org/10. 3390/f7030061

Forsman M, Holmgren J, Olofsson K (2016b) Tree stem diameter estimation from mobile laser scanning using line-wise intensity-based clustering. Forests 7: 206. https://doi.org/10.3390/f7090206

Hapca Al, Mothe F, Leban J-M (2007) A digital photographic method for 3D reconstruction of standing tree shape. Ann Forest Sci 64:631-637. https://doi. org/10.1051/forest:2007041

Hyyppä J, Virtanen J-P, Jaakkola A, Yu X, Hyyppä H, Liang X (2017) Feasibility of Google tango and Kinect for crowdsourcing forestry information. Forests 9:6. https://doi.org/10.3390/ 99010006

Jaakkola A, Hyyppä J, Kukko A, Yu X, Kaartinen H, Lehtomäki M, Lin Y (2010) A low-cost multi-sensoral mobile mapping system and its feasibility for tree measurements. ISPRS J Photogramm Remote Sens 65:514-522. https://doi. org/10.1016/j.isprsjprs.2010.08.002

Jaakkola A, Hyyppä J, Yu X, Kukko A, Kaartinen H, Liang X, Hyyppä H, Wang Y (2017) Autonomous collection of Forest field reference-the outlook and a first step with UAV laser scanning. Remote Sens 9:785. https://doi.org/10. 3390/rs9080785

Juraj Č, Ján T, Milan K, Martin M (2017) Estimation of diameter at breast height from mobile laser scanning data collected under a heavy forest canopy. J Forest Sci 63:433-441. https://doi.org/10.17221/28/2017-JFS

Kalliovirta J, Laasasenaho J, Kangas A (2005) Evaluation of the laser-relascope. For Ecol Manag 204:181-194. https://doi.org/10.1016/j.foreco.2004.09.020

Kukko A, Kaijaluoto R, Kaartinen H, Lehtola W, Jaakkola A, Hyyppä J (2017) Graph SLAM correction for single scanner MLS forest data under boreal forest canopy. ISPRS J Photogramm Remote Sens 132:199-209. https://doi.org/10. 1016/j.isprsjprs.2017.09.006

Liang X, Hyyppä J, Kaartinen H, Lehtomäki M, Pyörälä J, Pfeifer N, Holopainen M, Brolly G, Francesco P, Hackenberg J, Huang H, Jo H-W, Katoh M, Liu L, Mokroš M, Morel J, Olofsson K, Poveda-Lopez J, Trochta J, Wang D, Wang J, Xi Z, Yang B, Zheng G, Kankare V, Luoma V, Yu X, Chen L, Vastaranta M, Saarinen N, Wang Y (2018a) International benchmarking of terrestrial laser scanning approaches for forest inventories. ISPRS J Photogramm Remote Sens 144:137-179. https://doi.org/10.1016/j.isprs.jprs.2018.06.021

Liang X, Hyyppa J, Kukko A, Kaartinen H, Jaakkola A, Yu X (2014) The use of a mobile laser scanning system for mapping large forest plots. IEEE Geosci Remote Sens Lett 11:1504-1508. https://doi.org/10.1109/LGRS.2013.2297418

Liang X, Kukko A, Hyyppä J, Lehtomäki M, Pyörälä J, Yu X, Kaartinen H, Jaakkola A, Wang $Y(2018 b)$ In-situ measurements from mobile platforms: an emerging approach to address the old challenges associated with forest inventories. ISPRS J Photogramm Remote Sens. https://doi.org/10.1016/j.isprs.jprs.2018.04.019

Liang X, Wang Y, Jaakkola A, Kukko A, Kaartinen H, Hyyppä J, Honkavaara E, Liu J (2015) Forest data collection using terrestrial image-based point clouds from a handheld camera compared to terrestrial and personal laser scanning. IEEE 
Transact Geosci Remote Sens 53:5117-5132. https://doi.org/10.1109/TGRS. 2015.2417316

Luo H, Wang C, Wen C, Chen Z, Zai D, Yu Y, Li J (2018) Semantic labeling of mobile LiDAR point clouds via active learning and higher order MRF. IEEE Transact Geosci Remote Sens 56:3631-3644. https://doi.org/10.1109/TGRS. 2018.2802935

Marselis SM, Yebra M, Jovanovic T, van Dijk AIJM (2016) Deriving comprehensive forest structure information from mobile laser scanning observations using automated point cloud classification. Environm Model Software 82:142-151. https://doi.org/10.1016/j.envsoft.2016.04.025

Melkas T, Vastaranta M, Holopainen M, Hill R, Rosette J, Suárez J (2008) Accuracy and efficiency of the laser-camera. SilviLaser 2008 organizing committee, pp 315-324

Mokroš M, Liang X, Surový P, Valent P, Čerñava J, Chudý F, Tunák D, Saloň Š, Merganič J (2018) Evaluation of close-range photogrammetry image collection methods for estimating tree diameters. ISPRS Intl J Geo-Inform 7: 93. https://doi.org/10.3390/ijgi7030093

Oveland I, Hauglin M, Gobakken T, Næsset E, Maalen-Johansen I (2017) Automatic estimation of tree position and stem diameter using a moving terrestrial laser scanner. Remote Sens 9:350. https://doi.org/10.3390/rs9040350

Pyörälä J, Liang X, Saarinen N, Kankare V, Wang Y, Holopainen M, Hyyppä J, Vastaranta M (2019) Assessing branching structure for biomass and wood quality estimation using terrestrial laser scanning point clouds. Can J Remote Sens:1-14. https://doi.org/10.1080/07038992.2018.1557040

Pyörälä J, Liang X, Vastaranta M, Saarinen N, Kankare V, Wang Y, Holopainen M, Hyyppä J (2018) Quantitative assessment of scots pine (Pinus Sylvestris L.) whorl structure in a Forest environment using terrestrial laser scanning. IEEE J Select Topics Appl Earth Observ Remote Sens 11:3598-3607. https://doi. org/10.1109/JSTARS.2018.2819598

Repola J (2009) Biomass equations for scots pine and Norway spruce in Finland. Silv Fenn 43:625-647

Suomalainen J, Anders N, labal S, Roerink G, Franke J, Wenting P, Hünniger D, Bartholomeus H, Becker R, Kooistra L (2014) A lightweight hyperspectral mapping system and photogrammetric processing chain for unmanned aerial vehicles. Remote Sens 6:1 1013-11030. https://doi.org/10.3390/rs61111013

Tomaštík J, Saloň Š, Tunák D, Chudý F, Kardoš M (2017) Tango in forests - an initial experience of the use of the new Google technology in connection with forest inventory tasks. Comput Electron Agric 141:109-117. https://doi. org/10.1016/j.compag.2017.07.015

Wallace L, Lucieer A, Watson C, Turner D (2012) Development of a UAV-LiDAR system with application to forest inventory. Remote Sens 4:1519-1543. https://doi.org/10.3390/rs4061519

Wallace L, Musk R, Lucieer A (2014) An assessment of the repeatability of automatic forest inventory metrics derived from UAV-borne laser scanning data. IEEE Transact Geosc Remote Sens 52:7160-7169. https://doi.org/10. 1109/TGRS.2014.2308208

Wang Y, Hyyppä J, Liang X, Kaartinen H, Yu X, Lindberg E, Holmgren J, Qin Y, Mallet C, Ferraz A, Torabzadeh H, Morsdorf F, Zhu L, Liu J, Alho P (2016) International benchmarking of the individual tree detection methods for modeling 3-D canopy structure for Silviculture and Forest ecology using airborne laser scanning. IEEE Transact Geosci Remote Sens 54:5011-5027. https://doi.org/10.1109/TGRS.2016.2543225

Wang Y, Lehtomäki M, Liang X, Pyörälä J, Kukko A, Jaakkola A, Liu J, Feng Z, Chen R, Hyyppä J (2019) Is field-measured tree height as reliable as believed - a comparison study of tree height estimates from field measurement, airborne laser scanning and terrestrial laser scanning in a boreal forest. ISPRS J Photogramm Remote Sens 147:132-145. https://doi.org/10.1016/j.isprsjprs.2018.11.008

Wieser M, Mandlburger G, Hollaus M, Otepka J, Glira P, Pfeifer N (2017) A case study of UAS borne laser scanning for measurement of tree stem diameter. Remote Sens 9:1154. https://doi.org/10.3390/rs9111154

\section{Submit your manuscript to a SpringerOpen ${ }^{\circ}$ journal and benefit from:}

- Convenient online submission

- Rigorous peer review

- Open access: articles freely available online

High visibility within the field

- Retaining the copyright to your article

Submit your next manuscript at $\boldsymbol{\nabla}$ springeropen.com 